\title{
To know the Indicators and Predictors of Zonulopathy can Prevent the Headache of Eye Surgeon
}

\section{Burak Turgut*}

Department of Ophthalmology, Fırat University School of Medicine, Elazig, Turkey

${ }^{*}$ Corresponding author: Burak Turgut, Associate Professor of Ophthalmology, Department of Ophthalmology, Firat University School of Medicine, Elazig, Turkey, Tel: +904242333555; Fax: +904242388096; E-mail: drburakturgut@gmail.com

Received date: May 20, 2017; Accepted date: May 26, 2017; Published date: May 30, 2017

Citation: Turgut B (2017) To know the Indicators and Predictors of Zonulopathy can Prevent the Headache of Eye Surgeon. J Eye Cataract Surg Vol 3 Iss 2: 30 .

Copyright: (c) 2017 Turgut B. This is an open-access article distributed under the terms of the Creative Commons Attribution License, which permits unrestricted use, distribution, and reproduction in any medium, provided the original author and source are credited.

\section{Editorial}

The cataract is the opacity in the crystalline lens and it is the leading cause of blindness over the world. Cataract surgery is currently the unique treatment for cataract. Cataract surgery is the most commonly performed type of ocular surgery over the world. Although phacoemulsification is a secure surgical type for cataract removal with a success rate of over $98 \%$, it can result in serious complications [1].

The zonule of Zinn (the suspensory ligaments of the lens, Zinn's membrane, ciliary zonule) is a ring of fibrous fibers connecting the ciliary body with the crystalline lens. It originates from the basal laminae of the non-pigmented epithelium of the ciliary body, and adhere the lens capsule at the equatorial region. Zonules provide to remain the holding position of the lens and it plays the critical role in accommodation. Zonules are mainly made of fibrillin, a connective tissue glycoprotein that provides strength and elasticity to the lens fibers. As fibrillin is coded by the FBN1 gene, the mutations in this gene can cause zonulopathy [2].

Zonulopathy is a clinical entity describing the deficiency of zonular support of the lenticular capsule. Zonulopathy can occur due to the weakness, dehiscence or dialysis or fragility or instability or laxity of the zonules in congenital, genetic or acquired forms. It has been reported that a percent of approximately $5 \%$ of zonulopathy cases is recognizable at the time of surgery and that zonular dehiscence develops commonly at the stage of the removal of the lens nucleus or cortical material in cataract surgery [3]. The common causes of zonulopathy are pseudoexfoliation syndrome, zonulolysis due to ocular trauma, iatrogenic zonulolysis due to previous ocular interventions damaging to the zonules such as lens surgery, pars plana vitrectomy, repeated intravitreal injections, and various ocular disorders including high myopia, spherophakia, retinopathy of prematurity, ultra-brunescent cataracts, retinitis pigmentosa, aniridia, advanced age and intraocular tumor. Additionally, systemic diseases or syndromes that affect the zonules or fibrillin such as porphyria, hyperlysinemia, Marfan syndrome, homocystinuria, scleroderma, Weil-Marchesani syndrome and Ehlers-Danlos syndrome may cause the zonulopathy [4-7]. If the cause of zonulopathy is a specific disease such as pseudoexfoliation syndrome affecting the strength of the zonular system, the zonular fibers are more fragile and they are four times more likely to break [3,5,7-9].

Preoperative signs of zonulopathy are phacodonesis/pseudophacodonesis, iridodonesis, a tilted or subluxated or eccentric lens, the presence of a shallow or hyper deep anterior chamber in an eye, asymmetric axial anterior chamber or angle depth between both eyes, a space between iris and lens (iridolenticular gap) and visible lens equator [8,9].

Intraoperative signs of zonulopathy include difficulty in the central perforation of the anterior lenticular capsule with the cystotome, extremely wrinkling and the mobility of the anterior lenticular capsule, and heavy movement of the peripheral lenticular capsule when capsular flap is maneuvered with capsule forceps (pseudoelasticity), the visualization of the peripheral capsule fornix, prominent anterior capsule stria (trampolining) during anterior capsulorhexis, eccentric nucleus displacement with difficult nucleus rotation, difficulty in the cortex removal with posterior capsular stria and infolding of the peripheral capsule during irrigation/aspiration of cortex, vitreal prolapse into the anterior chamber, sudden deepening of the anterior chamber during phacoemulsification [8,9]. Zonulopathy can cause the lens subluxation, vitreous prolapsus and consequently trigger the complications such as cystoid macular edema, retinal tears and other serious retinal or choroidal complications $[3,5,6,10,11]$.

The surgeon should look for above mentioned preoperative signs, indicators and predictors of zonulopathy before the surgery and should perform the planning and preparation for the management of possible zonulopathy related complications. If the surgeon experiences the zonulopathy with the observation of intraoperative signs and indicators during operation, she/he should know how to deal with complications. To describe the predictors and indicators that could indicate zonulopathy would be useful for the eye surgeon because this would help in the selection of appropriate devices and surgical approach for ocular surgery. Additionally, this will be able to prevent the headache of an eye surgeon. 


\section{References}

1. Chen JL, Chen A (2017) Astronomy for Older Eyes: A Guide for Aging Backyard Astronomers Springer International Publishing AG, pp. 44.

2. Kaufman PL, Alm A (2010) Adler's physiology of the eye (11th edn.). St. Louis, Mo: Mosby pp. 145-146.

3. Guzek JP, Holm M, Cotter J (1987) Risk factors for intraoperative complications in 1000 extracapsular cataract cases. Ophthalmology 94: 461-466.

4. Dureau P (2008) Pathophysiology of zonular diseases. Curr Opin Ophthalmol 19: 27-30.

5. Shingleton BJ, Crandall AS, Ahmed II (2009) Pseudoexfoliation and the cataract surgeon: preoperative, intraoperative, and postoperative issues related to intraocular pressure, cataract, and intraocular lenses. J Cataract Refract Surg 35: 1101-1120.

6. Küchle $M$, Viestenz $A$, Martus $P$, Händel $A$, Jünemann $A$, et al. (2000) Anterior chamber depth and complications during cataract surgery in eyes with pseudoexfoliation syndrome. Am J Ophthalmol 129: 281-285.
7. Naumann GO, Schlötzer-Schrehardt U, Küchle M (1998) Pseudoexfoliation syndrome for the comprehensive ophthalmologist. Intraocular and systemic manifestations. Ophthalmology 105: 951-968.

8. Yaguchi S, Yaguchi S, Asano Y, Aoki S, Hamakawa M, et al. (2015) Categorization and Surgical Techniques of Weak Zonule Based on Findings at Capsulorhexis during Cataract Surgery. J Clin Exp Ophthalmol 6: 407.

9. Shingleton BJ, Neo YN, Cvintal V, Shaikh AM, Liberman P, et al. (2017) Outcome of phacoemulsification and intraocular lens implantion in eyes with pseudoexfoliation and weak zonules. Acta Ophthalmol 95: 182-187.

10. Wilson DJ, Jaeger MJ, Green WR (1987) Effects of extracapsular cataract extraction on the lens zonules. Ophthalmology 94: 467-470.

11. Skuta GL (1987) Zonular dialysis during extracapsular cataract extraction in pseudoexfoliation syndrome. Arch Ophthalmol 105: 632-634. 